\title{
Niveles de nitrógeno en suelos del cantón Chambo, provincia de Chimborazo
}

\author{
Nitrogen levels in soils of Chambo canton, province of Chimborazo
}

\author{
Yánez-Yánez Wilfrido ${ }^{1 *}$, Núñez-Torres Oscar Patricio ${ }^{1}$, Yánez-Borja Darwin Benigno², \\ Rivera-Guerra Verónica Elizabeth ${ }^{1}$, López-Villacís Isabel Cristina ${ }^{1}$, Velástegui-Espín Giovanny Patricio ${ }^{1}$
}

\begin{tabular}{l}
\hline Datos del Artículo \\
\hline 1Facultad de Ciencias Agropecuarias \\
Universidad Técnica de Ambato, Tungu- \\
rahua, Ecuador. \\
Casilla postal: 18-01-334. \\
Telf: (+593) 032746151- 0984388229. \\
op.nuñez@uta.edu.ec \\
ve.rivera@uta.edu.ec \\
ic.lopez@uta.edu.ec \\
gp.velastegui@uta.edu.ec \\
2Centro de levantamientos integrados de \\
recursos naturales por sensores remotos \\
(Clirsen). Km 4, al norte de Latacunga y a \\
44 Km. al sur de Quito. \\
Telf: (+593) 042 2645241-022 543193. \\
darwinbenivanez@vahoo.es \\
*Dirección de contacto: \\
Wilfrido Yánez-Yánez \\
Facultad de Ciencias Agropecuarias \\
Universidad Técnica de Ambato, Tungu- \\
rahua, Ecuador. \\
Casilla postal: 18-01-334. \\
Telf: (+593) 032746151- 0984388229 \\
E-mail: aw.vanez@uta.edu.ec \\
\hline Palabras clave: \\
\hline Precipitación, \\
textura, \\
macronutriente, \\
información geopedológica, \\
pH, \\
erodado, \\
USLE. \\
\hline
\end{tabular}

J Selva Andina Biosph. 2017; 5(2):152-159.

\section{Historial del artículo.}

Recibido marzo, 2017

Devuelto agosto 2017

Aceptado septiembre, 2017.

Disponible en línea, noviembre 2017.

Editado por:

Selva Andina

Research Society

\section{Key words:}

Precipitation,

texture,

macronutrient,

geopedological information,

$\mathrm{pH}$,

eroded,

USLE.

\section{Resumen}

El objeto de este análisis es estimar la distribución del nitrógeno en suelos identificados en el cantón Chambo de la provincia de Chimborazo, se contó con información del proyecto "Generación de Geoinfomación para la gestión del Territorio a nivel Nacional” se tomó los siguientes parámetros: $\mathrm{pH}$, textura, pendiente, temperatura, nitrógeno y precipitación, se empleó el modelo USLE/RUSLE (Ecuación Universal de pérdida de Suelo/Ecuación Universal de Pérdida de Suelo Revisada), que es un lenguaje estructurado de consulta. Los suelos corresponden en su mayor parte al orden de los Andisoles, y en menor porcentaje a Molisoles e Inceptisoles, en pendientes variadas, con relieves desde muy suaves a muy fuertes. Los resultados revelan que mayores pérdidas de $\mathrm{N}$ se tienen cuando la precipitación es alta y la pendiente es muy pronunciada y la textura es franco arenoso. Se concluye que cantidades altas de nitrógeno se identificaron en suelos de texturas francas, con $\mathrm{pH}$ prácticamente neutro y con precipitaciones del orden de los $1800 \mathrm{~mm}$, en relieves ondulados, bajos contenidos de nitrógeno se detectaron en suelos de texturas franco arenoso, con $\mathrm{pH}$ medianamente ácido a ácido y en precipitaciones menores a $1800 \mathrm{~mm}$ con relieves muy fuertes a escarpados. Los diferentes niveles de nitrógeno tienen mucha relación con la textura, pendiente del terreno y la precipitación.

(C) 2017. Journal of the Selva Andina Biosph. Bolivia. Todos los derechos reservados.

Abstract

The objective of this analysis is to estimate the nitrogen distribution in soils identified in the Chambo canton of the province of Chimborazo, for which information was gathered by the project "Generation of Geoinformation for the Management of the Territory at National Level" of the That the following parameters were taken: $\mathrm{pH}$, texture, slope, temperature, nitrogen and precipitation, the USLE / RUSLE (Universal Soil Loss / Universal Soil Equation Revised Equation) model was used, which is a structured query language. The soils correspond mostly to the order of Andisols, and to a lesser extent to Molisoles and Inceptisols, on very varied slopes, with reliefs from very soft to very strong. The results reveal that higher losses of $\mathrm{N}$ are obtained when precipitation is high, the slope is very pronounced, and the texture is sandy loam. It is concluded that high amounts of nitrogen were identified in soils of free textures, with practically neutral $\mathrm{pH}$ and precipitations of the order of $1800 \mathrm{~mm}$, in undulating reliefs, low nitrogen contents were detected in sandy loam soils with medium acidic $\mathrm{pH}$ to acid and in precipitations smaller than $1800 \mathrm{~mm}$ with very strong reliefs to steep ones. Different levels of nitrogen have a lot to do with texture, slope and precipitation. 


\section{Introducción}

El suelo es un recurso natural no renovable, es decir, la tasa de regeneración está infinitamente por debajo de la tasa o ritmo al que se destruye (Moncayo 2012). Este fenómeno avanza de forma alarmante en el Ecuador observándose a lo largo del Callejón Interandino extensas áreas improductivas (INIAP 1993). Los factores que intervienen en este proceso son el agua, viento y actividades antrópicas (García \& Dorronsoro 2000), sin embargo, el agua es uno de los principales agentes erosivos (YánezYanez 2016), causando la lixiviación de nutrientes y perdida de su fertilidad (Montagnini \& Jordan 2002). El hombre, al realizar prácticas inadecuadas de manejo de suelo, agua, cultivos, destrucción de la cobertura vegetal, entre otros, ha contribuido a que se pierda la fertilidad rápida del suelo (INIAP 2003c). Si bien se ejecutan trabajos de conservación, estos han sido orientados a terrenos sumamente erosionados, mientras se han subestimado los efectos imperceptibles causados por el agua (YánezYanez 2016).

Con base a lo anterior es indispensable fortalecer los sistemas agrícolas sostenibles mediante el estudio de los diversos componentes que lo integran y la funcionalidad de los mismos (Barea 1991). Entre estos, es de suma importancia el estudio de macronutrientes como el nitrógeno, que resulta de la descomposición de residuos de plantas y animales en el suelo, que mediante un proceso biológico convierte el nitrógeno $(\mathrm{N})$ en nitrógeno disponible mediante su transformación a amonio y nitrato (Benzing 2001). Los cuales deben mantener su relación (N$\mathrm{NH}_{4} / \mathrm{N}^{-\mathrm{NO}_{3}}$ ), sin embargo, si las condiciones de suelo y clima lo permiten es posible emplear cantidades relativamente altas de $\mathrm{N}-\mathrm{NH}_{4}$ en relación a N$\mathrm{NO}_{3}$ (Villareal-Romero et al. 2002). Este proceso de 153 mineralización es indispensable durante el crecimiento de los cultivos ya que contribuye a la nutrición de los mismos (Echeverría et al. 2000).

La mayor parte del $\mathrm{N}$ del planeta Tierra se encuentra en las rocas ígneas de la corteza y el manto, sin embargo, esa forma de $\mathrm{N}$ no está disponible para las plantas, por lo menos en el mediano plazo. Por lo tanto, se puede considerar que esta forma del $\mathrm{N}$ no está disponible para los seres vivos.

En la naturaleza existen dos fuentes principales de reserva de $\mathrm{N}$ para las plantas, la mayor es la atmósfera, $78 \%$ del aire es $\mathrm{N}$, la otra reserva importante de $\mathrm{N}$ es la materia orgánica del suelo (MOS). Del total del $\mathrm{N}$ que hay en el suelo, aproximadamente el 98\% se encuentra formando compuestos orgánicos (García-Fayos 2004).

La característica más preciada de un suelo es su fertilidad, dándole la capacidad para sustentar de manera natural la vida. La pérdida de suelo fértil ocurre de manera natural debido a la erosión por agentes como el agua y el viento. La erosión del suelo reduce su fertilidad porque provoca la pérdida de minerales y materia orgánica de la capa más superficial del mismo, esto ocurre especialmente en terrenos secos y sin vegetación (Ibáñez 2008).

Por lo antes mencionado la presente investigación se ha formulado como objetivo de analizar la distribución del nitrógeno $(\mathrm{N})$ en los suelos del cantón Chambo, perteneciente a la Provincia de Chimborazo-Ecuador.

\section{Materiales y métodos}

La zona de análisis tiene una superficie de 16453.82 ha, en el Cantón Chambo, Provincia de Chimborazo, ubicada a $8 \mathrm{Km}$. de la cuidad de Riobamba, su 
altitud va desde los 2400 a $4730 \mathrm{msnm}$, zona de gran desarrollo agropecuario, teniendo como limitante principal la perdida de nutrientes (principalmente nitrógeno) por lixiviación, altas pendientes y la susceptibilidad al peligro de la erosión pluvial, abarca tres pisos climáticos: i) templado sub-andino, ii) frío andino y iii) glacial, tierras ocupadas en su mayor parte por suelos derivados de materiales volcánicos (piroclastos) caracterizados por ser de color oscuro, con niveles altos de materia orgánica, de texturas francas a franco limosas, con moderada a alta capacidad de retención de agua, generalmente profundos y en ocasiones poco profundos. En menor porcentaje aparecen suelos Molisoles e Inceptisoles caracterizados los primeros por ser profundos, de color oscuro, ricos en bases de cambio, de consistencia y estructura favorable para el desarrollo radicular de las plantas, con altos contenidos de materia orgánica, de texturas franco limosas a franco arcillo limosas, bien drenados. Los Inceptisoles son menos oscuros, de texturas francas a franco arcillo limosas y franco arcillosas, bien drenados. Estos suelos se ubican en relieves planos a muy fuertes con pendientes entre 2-5 hasta 70-100 \%. Por lo general son suelos aptos para el desarrollo de actividades agropecuarias; el limitante principal en algunas ocasiones es la pendiente y la susceptibilidad al peligro de la erosión pluvial y en otras el clima frío.

Para el desarrollo del análisis propuesto se tomó como base la información levantada para el Proyecto "Generación de Geoinformación para la gestión del Territorio a nivel Nacional". Además se conto con la versión digital del mapa geopedológico del cantón Chambo relacionada con: $\mathrm{pH}$, textura, pendiente, temperatura, nitrógeno y precipitación. Se empleo el modelo USLE, que es un lenguaje estructurado de consulta que permite estimar la distribución del nitrógeno en los suelos del cantón, que por ende repercute en nivel de fertilidad de los mismos. Las variables de análisis son las siguientes:

Pendiente. La pendiente del terreno afecta los escurrimientos superficiales imprimiéndoles velocidad. El tamaño de las partículas, así como la cantidad de material que el escurrimiento puede llevar en suspensión, son en función de la velocidad con la que el agua fluye sobre la superficie (Loredo-Osti et al. 2007) Tabla 1.

Tabla 1 Índice de calificación de pendientes

\begin{tabular}{cc}
\hline Etiqueta & Índice \\
\hline Plana 0 a 2\% $\%$ & 1 \\
Muy suave 2 a $5 \%$ & 1 \\
Suave 5 a $12 \%$ & 1 \\
Media 12 a $25 \%$ & 2 \\
Media a fuerte 25 a $40 \%$ & 2 \\
Fuerte 40 a $70 \%$ & 2 \\
Muy fuerte 70 a $100 \%$ & 3 \\
Escarpada 100 a $150 \%$ & 3 \\
No aplica & NA
\end{tabular}

Textura superficial. La textura es una propiedad relacionada con la erosividad, es así que si se tiene suelos de textura arenosa (alta porosidad) con presencia de lluvias que no alcancen cierta intensidad, (Tabla 2) absorberá toda el agua que reciba y por consiguiente en ausencia de escorrentía no existirá erosión, pero, por otro lado, al poseer baja proporción de arcilla existe poca unión de las partículas y por ello la escorrentía arrastrará el suelo.

Potencial hidrógeno $(p H)$. El $\mathrm{pH}$ hace referencia a la concentración en forma logarítmica de iones $\mathrm{H}^{+}$ de una solución acuosa que se ha mantenido en contacto con el suelo en el tiempo suficiente para alcanzar el equilibrio (Thompson \& Troeh 1988).

La determinación del $\mathrm{pH}$ sirve de pauta para interpretar algunas características de los suelos relacionadas especialmente con sus propiedades ácidas o alcalinas (Tabla 3) y el funcionamiento general en 
cuanto a la utilización y solubilidad de los nutrientes del suelo (INIAP 2006).

Tabla 2 Índice de calificación de la textura superficial

\begin{tabular}{cl}
\hline Etiqueta & Índice \\
\hline Arcillas & 1 \\
Arcillo-arenoso & 1 \\
Arcillo-limoso & 1 \\
Franco arcilloso & 1 \\
Franco arcillo- & 1 \\
arenoso & 1 \\
Franco arcillo-limoso & 2 \\
Franco & 2 \\
Limoso & 2 \\
Franco arenoso & 2 \\
Franco limoso & 3 \\
Arena & 3 \\
Arena muy fina & 3 \\
Arena fina & 3 \\
Arena media & 3 \\
Arena gruesa & 3 \\
Areno francosa &
\end{tabular}

Tabla 3 Índice de calificación de pH

\begin{tabular}{cc}
\hline Etiqueta & Índice \\
\hline Neutro & 1 \\
Prácticamente neutro & 1 \\
Ligeramente ácido & 1 \\
Ácido & 2 \\
Medianamente ácido & 2 \\
Muy ácido & 2 \\
Ligeramente alcalino & 3 \\
Medianamente alcalino & 3 \\
Alcalino & 3 \\
\hline Clirsen-Magap (Sigagro). 2014 &
\end{tabular}

La mayoría de los suelos productivos fluctúan entre un $\mathrm{pH}$ de 4.0 a 9.0 sin embargo la mayor parte de los cultivos crecen mejor en rangos comprendidos entre 5.5 y 7.5. No obstante, cada cultivo requiere un determinado rango de $\mathrm{pH}$ para su óptimo crecimiento (INIAP 2008).

Régimen de Temperatura del Suelo. Es descrito por la temperatura media anual del suelo, las fluctuaciones estacionales promedio con respecto a la media y la gradiente de temperatura más caliente y más fría por estación dentro de la zona de enraizamiento, (Tabla 4) que es la zona con profundidad de $100 \mathrm{~cm}$ (FAO 2009).

\section{Tabla 4 Índice de calificación del régimen de tempera- tura del suelo

\begin{tabular}{cc}
\hline Etiqueta & Índice \\
\hline Isotérmico & 1 \\
Isomésico & 2 \\
Isofrígido & 3 \\
\hline Clirsen-Magap (Sigagro). 2014
\end{tabular}

Nitrógeno. El nitrógeno promueve el rápido crecimiento e incrementa el tamaño de las hojas en las plantas, este elemento afecta todos los parámetros que contribuyen al rendimiento, el color de la hoja es un indicador de $\mathrm{N}$ en la planta, está estrechamente relacionado con la tasa de fotosíntesis y producción del cultivo. Cuando se aplica suficiente $\mathrm{N}$ al cultivo se incrementa la demanda por otros macronutrientes como P y K (Fairhurst \& Witt 2002). Tabla 5.

\section{Tabla 5 Índice de calificación de Nitrógeno}

\begin{tabular}{cc}
\hline Etiqueta & Índice \\
\hline Alto & 1 \\
Medio & 2 \\
Bajo & 3 \\
\hline Clirsen-Magap (Sigagro). 2014
\end{tabular}

\section{Resultados}

La distribución del $\mathrm{N}$ se muestra en la (Figura 1), en la que se consideran aspectos como: suelos arenosos. En realidad, son suelos de fácil manejo, absorben agua rápidamente, y con una menor capacidad erosiva comparada con suelos limosos y arcillosos. Son suelos con baja retención de humedad de fertilidad baja y con una baja capacidad de almacenar 
nutrientes. Sin embargo, con un manejo apropiado y un buen régimen de lluvias o con sistemas de riego, estos suelos pueden tener una adecuada capacidad productiva. En términos de manejo agronómico, en estos suelos es recomendable: i) Sembrarlos con las primeras lluvias con el objetivo de aprovechar al máximo la precipitación acumulada. ii) Cubrirlos con los residuos del cultivo anterior los cuales reducen perdidas superficiales del agua y a largo plazo genera créditos en el suplemento de nutrientes. iii) Realizar las aplicaciones de nitrógeno fraccionadas evitando pérdidas por lixiviación. iv) Tener muy en cuenta deficiencias de micronutrientes especialmente si el pH es superior a 7.2.

En términos de densidad es aconsejable manejar poblaciones bajas y nunca superar las 70000 plantas por ha.

Suelos franco limosos. Estos suelos tienen mayor capacidad de almacenar nutrientes y agua comparado con los anteriores. Son suelos bien aireados, aunque pueden ser fácilmente erodados por el agua o el viento, se cuartean cuando han sido sobremeca- nizados y los contenidos de materia orgánica han sido agotados. En términos de manejo agronómico, en estos suelos es recomendables protegerlos contra la erosión realizándoles labranza de conservación y evitar prepararlos cuando tiene altos contenidos de humedad.

Suelos arcillosos o franco arcillosos. Son suelos con buena fertilidad y capacidad de almacenar nutrientes y agua, usualmente tienen altos contenidos de materia orgánica. Sin embargo son de difícil manejo agronómico, se compactan si se mecaniza muy húmedo o muy seco. Dependiendo de su posición en el paisaje y del régimen de lluvias, son erodables puesto que la infiltración superficial es lenta. En términos de manejo agronómico, es recomendable: Mecanizarlos teniendo en cuenta el punto plástico inferior. Evitar excesos de mecanización que pulvericen los agregados del suelo. Devolver la mayor cantidad de residuos con el objetivo de aumentar los contenidos de carbono y consecuentemente la actividad microbiana y las secreciones de glumalina que incrementa la agregación del suelo.

Figura 1 Distribución geográfica de $\mathrm{N}$ en suelos del cantón Chambo

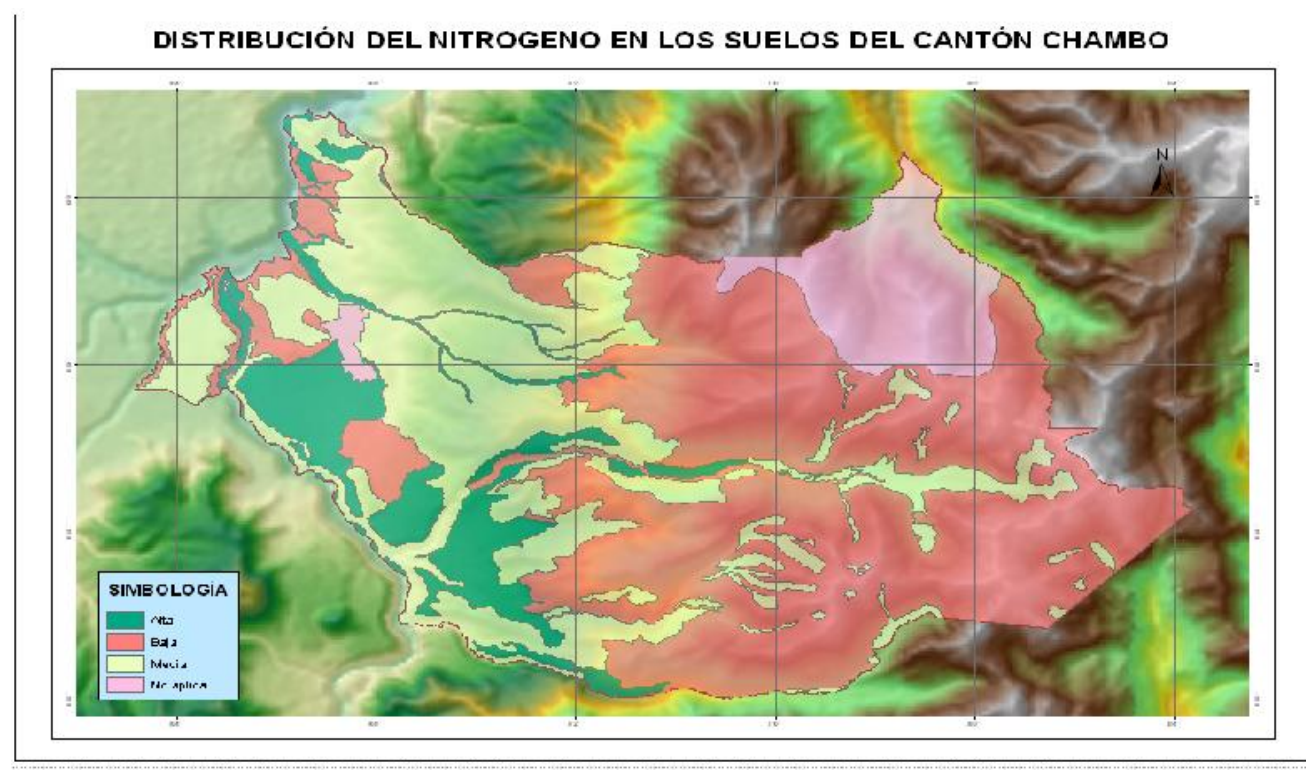


En el cantón Chambo se determinó contenidos altos de $\mathrm{N}$ en suelos de texturas francas, con $\mathrm{pH}$ prácticamente neutro, con precipitaciones que no sobrepasen los $1800 \mathrm{~mm}$ anuales, localizados en relieves planos hasta medianamente ondulados con pendientes de hasta $25 \%$, es así que a medida que aumenta la pendiente del terreno se produce mayor escorrentía y mayor erosión que, a su vez, será tanto más intensa cuando mayor sea la longitud del terreno en pendiente, como se observa en la Figura 1. Es necesario señalar que este rango de $\mathrm{N}$ se encuentra en mayor proporción en zonas destinadas a actividades agrícolas.

Contenidos medios de $\mathrm{N}$ se encontró en suelos de texturas franco arcillosas y franco limosas, de $\mathrm{pH}$ ligeramente ácido, con precipitaciones de 1100 a $1600 \mathrm{~mm}$ anuales, ubicados en relieves medios a fuertemente disectados con pendientes entre 25-40 $\%$ y $40-70 \%$ (Figura 1 ).

Bajos contenidos de $\mathrm{N}$ se identificaron en suelos de texturas franco arenoso (arena muy fina), $\mathrm{pH}$ medianamente ácido a ácido, con precipitaciones mayores a $1800 \mathrm{~mm}$ anuales, en relieves muy fuertemente disectados a escarpados con pendientes de 70-100 \% y más de $100 \%$. Las pérdidas de nitrógeno asimilable en el suelo se producen por los procesos siguientes:

\section{Discusión}

Inmovilización de los seres vivos. El nitrógeno inorgánico absorbido por los microorganismos y las plantas (y posteriormente por los animales) se convierte en nitrógeno orgánico (Oliveira et al. 2006), por un proceso inverso de la mineralización. El nitrógeno inmovilizado que no es exportado fuera del agrosistema vuelve al suelo con los residuos orgánicos (Fuentes-Yagüe 1999).

Fijación en algunas arcillas. El ion amonio tiene una gran afinidad por algunas arcillas (vermiculita, montmorillonita) y algunos iones adsorbidos pasan a ser entre las láminas de esas arcillas (fijación), donde permanecen durante más o menos tiempo, hasta que son liberados por efecto de otros iones más grandes o hidratados, que provocan la expansión de las arcillas. (Fuentes-Yagüe 1999).

El nitrógeno nítrico es muy soluble en el agua y no es retenido por el complejo coloidal del suelo, por lo que puede ser arrastrado en profundidad por el agua de percolación.

Las pérdidas por lixiviación son importantes cuando hay un movimiento descendente del agua y la actividad radical es escaza. Por ello se producen con mayor intensidad en suelos desnudos que en suelos cultivados y pueden ser de consideración en los inviernos lluviosos, en investigaciones realizadas por el INIAP se ha determinado que se pierde de 30 a $150 \mathrm{~kg} / \mathrm{ha}$ de $\mathrm{N}$ en suelos desnudos mientras que en suelos cultivados varía de 30 a $80 \mathrm{~kg} \mathrm{ha}^{-1}$.

Durante los periodos de sequía, una parte del nitrógeno lixiviado puede subir por capilaridad hasta la superficie. Ocurre generalmente cuando los cultivos están en un estado vegetativo avanzado, con el sistema radical bastante profundo.

El nitrógeno promueve el rápido crecimiento e incrementa el tamaño de las hojas en las plantas. El nitrógeno afecta todos los parámetros que contribuyen al rendimiento el color de la hoja es un indicador de $\mathrm{N}$ en la planta, está estrechamente relacionado con la tasa de fotosíntesis y producción del cultivo. Cuando se aplica suficiente $\mathrm{N}$ al cultivo se incrementa la demanda por otros macronutrientes como P y K. (Fairhurst \& Witt 2002). 
Cantidades altas de nitrógeno se identificó en suelos de texturas francas, con $\mathrm{pH}$ prácticamente neutro y con precipitaciones del orden de los $1800 \mathrm{~mm}$, en relieves ondulados.

Bajos contenidos de nitrógeno se detectaron en suelos de texturas francos arenosos, con $\mathrm{pH}$ medianamente ácido a ácido y en precipitaciones menores a $1800 \mathrm{~mm}$ con relieves muy fuertes a escarpados.

Los diferentes niveles de nitrógeno tienen mucha relación con la textura, pendiente del terreno y la precipitación.

Con un manejo adecuado del suelo, entendiéndose como buen manejo del suelo a aquel que asegura una adecuada nutrición, garantizando cosechas convenientes para la alimentación de la familia y para la generación de ingresos, la mejor manera de alimentar y proteger el suelo es aplicar regularmente materia orgánica o compost y mantenerlo cubierto con plantas. El sistema de cultivo en estratos es recomendable porque usa una mezcla de árboles y plantas de diferentes tiempos de maduración, lo que protege el suelo y recicla los nutrientes. Los cultivos de leguminosas (frijol, arveja, etc.) son muy útiles porque proveen una alimentación continua al suelo. Se logra mantener su fertilidad y estructura. (FAO 2016). Los suelos con elevada fertilidad aseguran cultivos con altos rendimientos, buena cobertura vegetal y como consecuencia, condiciones que permiten minimizar los efectos erosivos de la lluvia y el viento. Por estas razones, la fertilidad puede verse como aspecto clave en la conservación del suelo.

\section{Conflictos de intereses}

Esta investigación se la realizó en los suelos del cantón Chambo en la provincia de Chimborazo Ecuador y no presenta conflictos de interés.

\section{Agradecimientos}

Los autores agradecen al proyecto Generación de Geoinfomación para la gestión del Territorio a nivel Nacional, al GAD del cantón Chambo, y a la Universidad Técnica de Ambato, Facultad de Ciencias Agropecuarias, por el apoyo técnico, científico y logístico realizado a la presente investigación.

\section{Literatura citada}

Barea JM. Vesicular arbuscular mycorrhizae as modifiers of soil fertility, en Stewart BS. (ed). Advances in soil science. 1991; 15. Springer Verlag, Nueva York.

Benzing A. Agricultura Técnica. Fundamentos para la región andina. Agric Téc 2002;62(3): 485-6.

Clirsen-Magap (Sigagro). Proyecto de generación del mapa de cobertura y uso de la tierra del Ecuador Continental; 2013-2014.

Echeverría HE, San Martin NF, Bergonzi R. Métodos rápidos de estimación del nitrógeno potencialmente mineralizable en suelos. Cienc Suelo 2000;18(1):9-16.

Fairhust T, Witt C. Guía práctica para el manejo de nutrientes, Potash \& Phosphate Institute (PPI), Potash \& Phosphate Institute of Canada (PPIC); 2002. p. 55.

Organización de las Naciones Unidas para la Agricultura y la Alimentación. Guía para la descripción de suelos; 2009. $4^{\text {ed }}$. Roma. p. 99.

Organización de las Naciones Unidas para la Agricultura y la Alimentación. Manejo de Suelos. Hojas de información Básica; 2016. Roma. p. 9.

Fuentes-Yagüe JL. El suelo y los fertilizantes: Instituto nacional de reforma y desarrollo agrario 
Ediciones Mundi-Prensa, Madrid - España; 1999. p. 211.

García-Fayos P. Interacciones entre la vegetación y la erosión hídrica. En F. Valladares (ed.) Ecología del bosque mediterráneo en un mundo cambiante. Madrid: EGRAF; 2004. p. 309-34.

García I, Dorronsoro C. Contaminación del suelo. Dpto. de Edafología y Química Agrícola. Universidad de Granada sp; 2000.

Ibáñez JJ. Degradación del Suelo y Pérdida de Recursos Edáficos: Una Introducción. Fundación para el conocimiento; Madrid. 2008 [Citado enero 2017]. Disponible en: http://www.madri masd.org/blogs/universo/2008/09/17/101114.

Instituto Nacional Autónomo de Investigaciones Agropecuarias Metodologías de: Física de Suelos. Estación experimental Santa Catalina, departamento de manejo de suelos y aguas, Quito; 2006. p. 51.

Instituto Nacional Autónomo de Investigaciones Agropecuarias Metodologías de: Física de suelos. Quito, Estación Experimental Santa Catalina, Departamento de Manejo de Suelos y Aguas; 2008. p. 31-33.

Instituto Nacional Autónomo de Investigaciones Agropecuarias. La Erosión Hídrica: Proceso, Factores y Formas. Estación experimental Santa Catalina, departamento de suelos, área física y conservación de suelo y agua, Quito; 2003. BD 229.
Loredo-Osti C, Beltrán-López S, Moreno-Sánchez F, Casiano-Domínguez M. Predicción de riesgo a nivel microcuenca. Folleto técnico 29. INIFAP-CIRNE-Campo Experimental San Luís. San Luís Potosí; México. p. 2007.

Moncayo-Hurtado JM. Elaboración de un SIG agrícola con la ayuda de una aplicación Web .2012. Universidad San Francisco de Quito. Obtenido en: www.usfq.edu.com.

Montagnini F, Jordan CF. Reciclaje de nutrientes. En: Guariguata MR, Kattan GH, editores. Ecología y conservación de bosques neotropicales. Cartago (Costa Rica): Libro Universitario Regional; 2002. p. 167-92.

Oliveira Prendes JA, Afif-Khouri E, Mayor-López M. Análisis de suelos y plantas y recomendaciones de abonado, editores. Oviedo: Universidad de Oviedo; 2006. p. 146.

Thompson LM, Troeh FR. Los suelos y su fertilidad. $4^{\text {ed }}$. Barcelona ES, editorial Reverté SA; 1988. p. 661.

Villareal-Romero M, García-Estrada RS, OsunaEnciso T, Armenta-Bojorquez AD. Efecto de dosis y fuente de nitrógeno en rendimiento y calidad postcosecha de tomate en fertirriego. Terra. 2002;20:311-20.

Yánez-Yanez W. Manejo agroecológico de la fertilidad de los suelos; 2016. p. 51. 